\title{
Body Fat Mass is Better Indicator than Indirect Measurement Methods in Obese Children for Fatty Liver and Metabolic Syndrome
}

\author{
Masallah Baran ${ }^{a^{*}}$, Kivanc Celikkalkan ${ }^{b^{*}}$, Yeliz Cagan Appak ${ }^{\mathrm{c}}$, Miray Karakoyun ${ }^{\mathrm{d}}$, \\ Mehmet Bozkurt ${ }^{\mathrm{e}}$, Cemil Kocyigit ${ }^{\mathrm{f}}$, Ali Kanik ${ }^{\mathrm{g}}$, Bumin Nuri Dundar ${ }^{\mathrm{h}}$ \\ ${ }^{a}$ Department of Pediatric Gastroenterology Hepatology and Nutrition, Izmir Katip Celebi University School of Medicine, Izmir, Turkey. \\ ${ }^{b}$ Department of Pediatric, Tepecik Training and Research Hospital, Izmir, Turkey. \\ ${ }^{c}$ Department of Pediatric Gastroenterology Hepatology and Nutrition, Tepecik Training and Research Hospital, Izmir, Turkey. \\ ${ }^{d}$ Department of Pediatric Gastroenterology Hepatology and Nutrition, Ege University School of Medicine, Izmir, Turkey \\ ${ }^{e}$ Department of Radiology, Tepecik Training and Research Hospital, Izmir, Turkey \\ ${ }^{f}$ Department of Pediatric Endocrinology, Tepecik Training and Research Hospital, Izmir, Turkey \\ ${ }^{g}$ Department of Pediatric, Izmir Katip Celebi University School of Medicine, Izmir, Turkey \\ ${ }^{h}$ Department of Pediatric Endocrinology, Izmir Katip Celebi University School of Medicine, Izmir, Turkey.
}

Received 20 August 2019; Accepted 25 November 2019

\begin{abstract}
Introduction: To compare the bioelectric impedance analysis (BIA) with indirect measurement methods in the evaluation of obese children. To determine the diagnostic value of BIA in the fatty liver and metabolic syndrome (MS) in obese children. Population and methods: One hundred thirty-four obese children whom $\geqslant 10$ years of age were prospectively assessment. All patients were evaluated by foot to foot BIA and indirect measurement methods. Blood biochemical parameters such as glucose, lipids and insulin levels were studied and oral glucose tolerance test was performed. Fatty liver was assessed by ultrasonography. Compared BIA records and indirect measurements findings according to fatty liver and MS. Results: The study included females/males: 77/57, mean age of $13.3 \pm 2.2$ years. Fatty liver was detected in 94 patients, MS was diagnosed in 58 cases. There were no gender difference in terms of fatty liver and MS. Fatty liver was seen more frequently in patients with metabolic syndrome than in those without metabolic syndrome $(\mathrm{p}<0.001)$. Fat Mass $(\mathrm{FM})$ of $\geqslant 97$ th percentile was observed in $63 \%$ of the 94 patients with fatty liver versus $37.5 \%$ of 40 patients without fatty liver. A FM of $\geqslant 97$ th percentile was observed in $72 \%(n=42)$ of the 58 patients with metabolic syndrome, $42 \%(n=33)$ of 76 patients without MS. Body mass index, upper mid-arm circumference, waist circumference (WC), and hip circumference values were significantly increased in patients with fatty liver. There was a better correlation was determined between FM and FM Index with fatty liver compared to indirect measurement methods. BIA records were found moderately correlated with indirect measurements. Conclusion: Our results revealed that FM and FMI have a better correlated in obese children with fatty liver and metabolic syndrome than indirect measurement methods. The measurement of body FM by BIA can be used together with the indirect measurement methods to detect the fatty liver. FMI may be an alternative diagnostic criterion instead of WC for diagnosis of MS in children.

Keywords: Body Composition; Obesity; Children; Fatty Liver.
\end{abstract}

\section{Introduction}

Obesity, a disease in which an excessive storage of fat changes the body's metabolic balances, is increasing among children [1]. Body fat ratio is strongly associated with the risk of chronic diseases, such as non-alcoholic fatty liver,

* Corresponding author: mbaran2509@gmail.com; kivanccx@hotmail.com

\section{doi) http://dx.doi.org/10.28991/SciMedJ-2019-0104-2}

$>$ This is an open access article under the CC-BY license (https://creativecommons.org/licenses/by/4.0/).

(C) Authors retain all copyrights. 
insulin resistance, type II diabetes, dyslipidemia, hypertension (HT) and metabolic syndrome (MS) in childhood obesity $[2,3]$. In obese patients, the body fat ratio is measured using both direct [underwater weighing, bioelectric impedance analysis (BIA), computerized tomography, magnetic resonance imaging, dual-energy X-ray absorption (DEXA), and ultrasonography (USG)] and indirect methods include anthropometric measurements [Body mass index (BMI), upper mid-arm circumference (UMAC), waist circumference (WC), and hip circumference (HC)] [4-6]. Bioimpedance analysis was evaluated as a valuable alternative to DEXA for body fat measurement [7]. Bioelectric impedance analysis is a relatively simple, quick, noninvasive and relatively low cost method for estimating body composition compared to some of the other methods of body composition analysis. In this study, we aimed to compare the BIA with indirect measurement. There is no study investigating the relationship between body fat ratio by BIA with fatty liver and metabolic syndrome in obese children in the literature. This study is evaluation of body fat composition by BIA in the diagnosis of fatty liver and MS in obese children.

\section{Population and Methods}

Between November 2016 and June 2018, 134 obese children $\geq 10$ years of age were prospectively studied. This observational longitudinal study was approved by the local ethics committee (30.09.2014/18). Informed consent was obtained from the family.

In this study, children were defined as obese if they had a BMI $\left(\mathrm{kg} / \mathrm{m}^{2}\right)$ greater than those in the $95 \%$ percentile, which is based on the Turkish standard [8]. We used a BMI standard deviation score (SDS) for the statistical analysis.

\subsection{Indirect Methods (Anthropometric Measurements)}

All measurements were performed by the same doctor and were recorded in the study files. The weight of each participant was measured while participants were wearing a light robe and no shoes. The height of each patient was measured while standing upright and with the back and head in an upright position.

\subsection{Upper mid-arm Circumference Measurement}

The distance between the humeral head and olecranon projection was measured while the patient was standing, with the left arm flexed and the elbow pointed straight down. After the mid-arm line was determined, it was measured while the arm was deflexed.

\subsection{Waist Circumference Measurement}

The distance was measured between the last rib and the crista iliac while the patient was standing upright.

\subsection{Hip Circumference Measurement}

The widest diameter on the trochanter in the horizontal position was measured when the patient was standing with legs 20-30 cm apart.

\subsection{Fatty Liver Evaluation}

This test was performed by the same radiologist and using a B-mode USG method. Fatty infiltration was graded on a scale of $0-3$ as follows: no fatty infiltration, 0 ; mild infiltration, 1; medium infiltration, 2; and severe infiltration, 3 [9].

\subsection{Bioelectric Impedance Analysis, Body Fat Mass, and Weight Measurement}

These measurements were taken with a Tanita SC-331S Body Composition Analyzer (Tanita; Tanita Corp.) foot-tofoot (four electrodes, twist on each foot) system device with the patient undressed. Fat mass ( $\mathrm{kg}$ and \%), muscle mass $\left(\mathrm{MM} ; \mathrm{kg}\right.$ and \%) values, and fat mass index (FMI; $\mathrm{kg} / \mathrm{m}^{2}$ ); Individual FMI was calculated using the formula: fat mass $(\mathrm{kg}) /$ height $^{2}$ [10]. Fat mass percentiles were evaluated based on the reference range for Turkish children [11].

\subsection{Laboratory Results}

All results of oral glucose tolerance tests (OGTT), as well as LDL, HDL, triglyceride, cholesterol, uric acid, transaminase, fasting blood glucose, and fasting plasma insulin levels, were recorded.

Impaired fasting glucose and type 2 diabetes mellitus were defined by OGTT results according to the American Diabetes Association guidelines [12].

\subsection{Metabolic Syndrome (MS)}

This term was defined according to the Modified International Diabetes Federation criteria for children and adolescents [13].

\subsection{Insulin Resistance}

The homeostasis model of assessment for insulin resistance (HOMA-IR) index was implemented using the following 
equation: fasting insulin concentration $(\mu \mathrm{U} / \mathrm{mL}) \times$ fasting glucose concentration $(\mathrm{mg} / \mathrm{dl}) / 22.5$. Prepubertal values of 2.5 and above, as well as pubertal values of 4.0 and above, were considered as insulin resistance [14].

\section{Statistical Analysis}

Parametric tests, was checked via Levene's test. The assumption of normality was tested via the Shapiro-Wilk test. To compare the differences between the two groups, the student's t-test was used and the Mann-Whitney U-test was used when these prerequisites were not fulfilled. Spearman's or Pearson's correlation coefficient was used to measure the relationship between two variables according to variable type. A chi-square test was used to determine the two discrete variables. A receiver operating characteristic (ROC) curve analysis was used to measure the discriminative power of the method. The data was evaluated via SPPS 20 (SPSS Statistics for Windows, Version 20.0., released in 2011, Armonk, NY: IBM Corp.), p < 0.05 was taken as the significance level.

\section{Results}

The study included 134 children (77 females, $57.5 \%$ ), mean age of the overall participants was $13.3 \pm 2.2$. There were no differences in terms of age between the two genders (F: $13.4 \pm 2.4$ years, M: $13.1 \pm 2.1$ years). BMI-SDS mean was $2.9 \pm 0.6$ in 134 cases. No difference was determined in the BMI-SDS based on gender $(\mathrm{p}=0.77)$.

An evaluation of USG results indicated that $70 \%$ of patients $(n=94)$ had fatty liver. The distribution is as follows: grade $1,36 \%(n=49)$; grade 2, $27 \%(n=36)$; and grade $3,6.7 \%(n=9)$. No difference was determined in the frequency of fatty liver based on gender $(\mathrm{p}=0.99)$.

When OGTT results were evaluated, no patient was diagnosed with diabetes mellitus, impaired fasting glucose in 24 cases and impaired glucose tolerance were detected in 24 cases. MS was found in 58 patients (43.3\%). No significant differences were detected between males and females with respect to the prevalence of MS $(p=0.9)$. Fatty liver was seen more frequently in patients with MS than in those without MS ( $\mathrm{p}<0.001)$.

BIA records; evaluation of the FM results determined that $56 \%$ of the patients $(n=75)$ were in the $\geq 97^{\text {th }}$ percentile. No difference was found percent of $\geq 97^{\text {th }} F M$ based on gender $(p=0.08)$. Fatty liver was determined $80 \%$ in the cases with $\geq 97^{\text {th }}$ percentile of FM $(n=75)$. FM of $\geq 97^{\text {th }}$ percentile was observed in $63 \%$ of the 94 patients with fatty liver versus $37.5 \%$ of 40 patients without fatty liver. A FM of $\geq 97^{\text {th }}$ percentile was observed in $72 \%$ ( $=42$ ) of the 58 patients with metabolic syndrome, $42 \%(n=33)$ of 76 patients without MS. FM and FMI significantly higher in cases with fatty liver and metabolic syndrome. Comparison of all measurements records according to fatty liver and MS were shown in Tables 1 and 2. Moderate correlations were determined between indirect measurements and BIA records in this study population (Tables 3 and 4).

Table 1. Demographic, indirect measurement methods and BIA findings in cases according to fatty liver

\begin{tabular}{cccc}
\hline Subjects & Fatty liver (n=94) & No Fatty liver $(\mathbf{n = 4 0})$ & p \\
\hline Sex (F/M) & $54 / 40$ & $23 / 17$ & 0.99 \\
Age & $14.1 \pm 2.2$ & $13 \pm 2$ & 0.006 \\
MS (n=58) & 54 & 4 & $<0.001$ \\
Triglycerides (mg/dL) & $152 \pm 69$ & $106 \pm 28$ & $<0.001$ \\
Cholesterol (mg/dL) & $178 \pm 36$ & $158 \pm 22$ & 0.001 \\
ALT (UI/L) & $34 \pm 27$ & $21 \pm 7$ & 0.002 \\
BMI (SDS) & $3.03 \pm 0.64$ & $2.07 \pm 0.52$ & 0.009 \\
UMAC (cm) & $31,6 \pm 3,3$ & $30,1 \pm 3,4$ & 0,018 \\
WC (cm) & $106,4 \pm 11,1$ & $101,2 \pm 9,6$ & 0,01 \\
HC (cm) & $108,8 \pm 13,2$ & $102,2 \pm 11,1$ & 0,01 \\
FM (kg) & $38,3 \pm 10,1$ & $31,3 \pm 7,2$ & $<0.001$ \\
FMI (kg/m $\left.{ }^{2}\right)$ & $19.2 \pm 0.3$ & $16.4 \pm 2.5$ & $<0.001$ \\
MM (kg) & $44.8 \pm 9,2$ & $45.4 \pm 12,7$ & 0.76 \\
FM/MM & $0.85 \pm 0,14$ & $0.7 \pm 0,15$ & $<0.001$ \\
\hline
\end{tabular}

BMI: Body mass index, SDS: Standard deviation score, UMAC: Upper mid-arm circumference, WC: Waist circumference, $H C$ : Hip circumference, FM: Fat mass, MM: Muscle mass, FMI: Fat mass index, $M S$ : Metabolic syndrome. 
Table 2. Demographic, indirect measurement methods and BIA findings in cases according to MS

\begin{tabular}{cccc}
\hline Subjects & MS (n=58) & No MS $(\mathbf{n}=\mathbf{7 6})$ & p \\
\hline Sex (F/M) & $54 / 40$ & $23 / 17$ & 0.99 \\
Age & $13.3 \pm 2$ & $13.2 \pm 2.2$ & 0.9 \\
Triglycerides (mg/dL) & $182 \pm 71$ & $105 \pm 28$ & $<0.001$ \\
HDL (mg/dL) & $36.8 \pm 7$ & $44 \pm 9$ & $<0.001$ \\
ALT (UI/L) & $36 \pm 30$ & $26.1 \pm 17$ & 0.019 \\
Fasting Insulin & $22.7 \pm 14.5$ & $19.2 \pm 10.7$ & 0.1 \\
BMI (SDS) & $3.09 \pm 0.66$ & $2.83 \pm 0.57$ & 0.015 \\
UMAC (cm) & $32,9 \pm 3,2$ & $30,1 \pm 3,3$ & $<0.001$ \\
WC (cm) & $108,9 \pm 10,9$ & $101.8 \pm 9,9$ & $<0.001$ \\
HC (cm) & $111,8 \pm 12,8$ & $103,3 \pm 11,7$ & $<0.001$ \\
FM (kg) & $40.6 \pm 9.7$ & $32,8 \pm 8.6$ & $<0.001$ \\
FMI (kg/m $\left.{ }^{2}\right)$ & $19.8 \pm 2.8$ & $17.3 \pm 2.9$ & $<0.001$ \\
MM $(\mathrm{kg})$ & $47.2 \pm 9,4$ & $43.2 \pm 10.8$ & 0.028 \\
FM/MM & $0.86 \pm 0,14$ & $0.76 \pm 0,16$ & 0.001 \\
\hline
\end{tabular}

$B M I$ : Body mass index, SDS: Standard deviation score, UMAC: Upper mid-arm circumference, WC: Waist circumference, $H C$ : Hip circumference, FM: Fat mass, MM: Muscle mass, FMI: Fat mass index, MS: Metabolic syndrome.

Table 3. Correlation coefficients of body measurements with fatty liver and MS

\begin{tabular}{ccc}
\hline Body Measurement & NAFL $(\mathbf{r} / \mathbf{p})$ & MS $(\mathbf{r} / \mathbf{p})$ \\
\hline WC & $0.229 / 0.008$ & $0.317 /<0.001$ \\
UMAC & $0.211 / 0.015$ & $0.308 /<0.001$ \\
HC & $0.231 / 0.007$ & $0.326 /<0.001$ \\
BMI (SDS) & $0.269 / 0.002$ & $0.248 / 0.04$ \\
FM & $0.317 /<0.001$ & $\mathbf{0 . 3 8 3} /<\mathbf{0 . 0 0 1}$ \\
FMI & $\mathbf{0 . 4 0 5 / < 0 . 0 0 1}$ & $0.349 /<0.001$ \\
FM/MM & $0.401 /<0.001$ & $0.287 / 0.001$ \\
\hline
\end{tabular}

$B M I$ : Body mass index, SDS: Standard deviation score, UMAC: Upper mid-arm circumference, $W C$ : Waist circumference, $H C$ : Hip circumference, $F M$ : Fat mass, MM: Muscle mass, FMI: Fat mass index, MS: Metabolic syndrome

Table 4. Correlation coefficients of indirect measurement methods and BIA records

\begin{tabular}{cccc}
\hline Body Size & FMI (r) & FM (r) & p \\
\hline WC & 0.645 & 0.795 & $<0.001$ \\
UMAC & 0.566 & 0.684 & $<0.001$ \\
HC & 0.652 & 0.807 & $<0,001$ \\
BMI (SDS) & 0.638 & 0.593 & $<0.001$ \\
\hline
\end{tabular}

BMI: Body mass index, SDS: Standard deviation score, UMAC: Upper mid-arm circumference, WC: Waist circumference, $H C$ : Hip circumference, $F M$ : Fat mass, MM: Muscle mass, FMI: Fat mass index, MS: Metabolic syndrome. Pearson correlation was used.

Laboratory findings; significant differences in serum triglycerides, HOMA-IR, and HDL values were observed in patients whose fatty liver and MS ( $\mathrm{p}<0.01)$. Comparison of laboratory findings was shown in Table I and Table II according to clinical picture. When evaluation of correlation plasma lipid and measurement methods FMI, FM was correlated better method with TG level $(\mathrm{r}=0.257, \mathrm{r}=0.326, \mathrm{p}=0.001)$ while no significant correlation between BMI and TG level. UMAC was the method that showed the better correlation with cholesterol in indirect measurement methods $(\mathrm{r}=0.237, \mathrm{p}=0.06)$. No significant correlation was found between cholesterol level and used all measurement methods $(\mathrm{p}>0.05)$.

ROC curve analysis was shown based on fatty liver and measurement methods (Figure 1). Analysis of ROC curve according to MS and measurement methods were shown in Figure 2. The cut-off value of the FMI was $13.2 \mathrm{~kg} / \mathrm{m}^{2}$, and the sensitivity of fatty liver was found to be $71 \%$ while the specificity was $68 \%(95 \% \mathrm{CI}=0.672-0.839)$. An evaluation of $\mathrm{HC}$, the cut-off value was $102.5 \mathrm{~cm}$, sensitivity was $71 \%$, and specificity was $50 \%$, (95\% CI $=0.546-0.745)$. Evaluation based on metabolic syndrome revealed, the cut-off value was $13.2 \mathrm{~kg} / \mathrm{m}^{2}$ for FMI, sensitivity was $76 \%$, and specificity was $53 \%(95 \% \mathrm{CI}=0.614-0.792)$ while WC cut-off value is $102.5 \mathrm{~cm}$, sensitivity was $72 \%$, and specificity 
was 54\% (95\% CI = 0.542-0.777, Figure 2). BMI-SDS cut-off level for MS was found 2.94, sensitivity was 72\%, and specificity was $53 \%$, while cut-off level for fatty liver was found 2.79 , sensitivity $72 \%$, specificity $53 \%$ was determined.

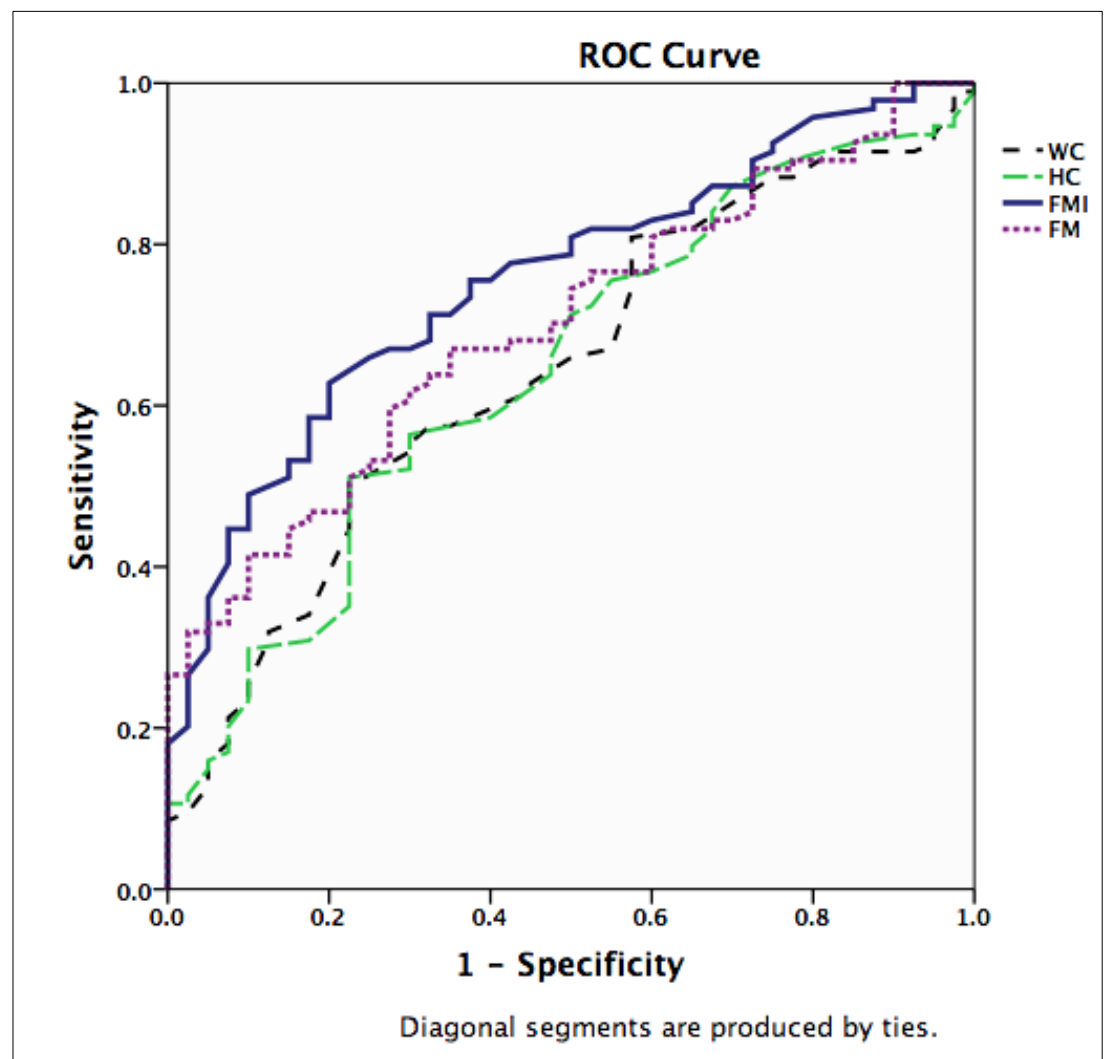

Area under the Curve: FMI: 0.755, FM: 0.705, WC: 0.645, HC: 0.645 .

WC: Waist circumference, HC: Hip circumference, FM: Fat mass, FMI: Fat mass index.

Figure 1. ROC curve analysis of fat mass, fat mass index and indirect methods according to fatty liver

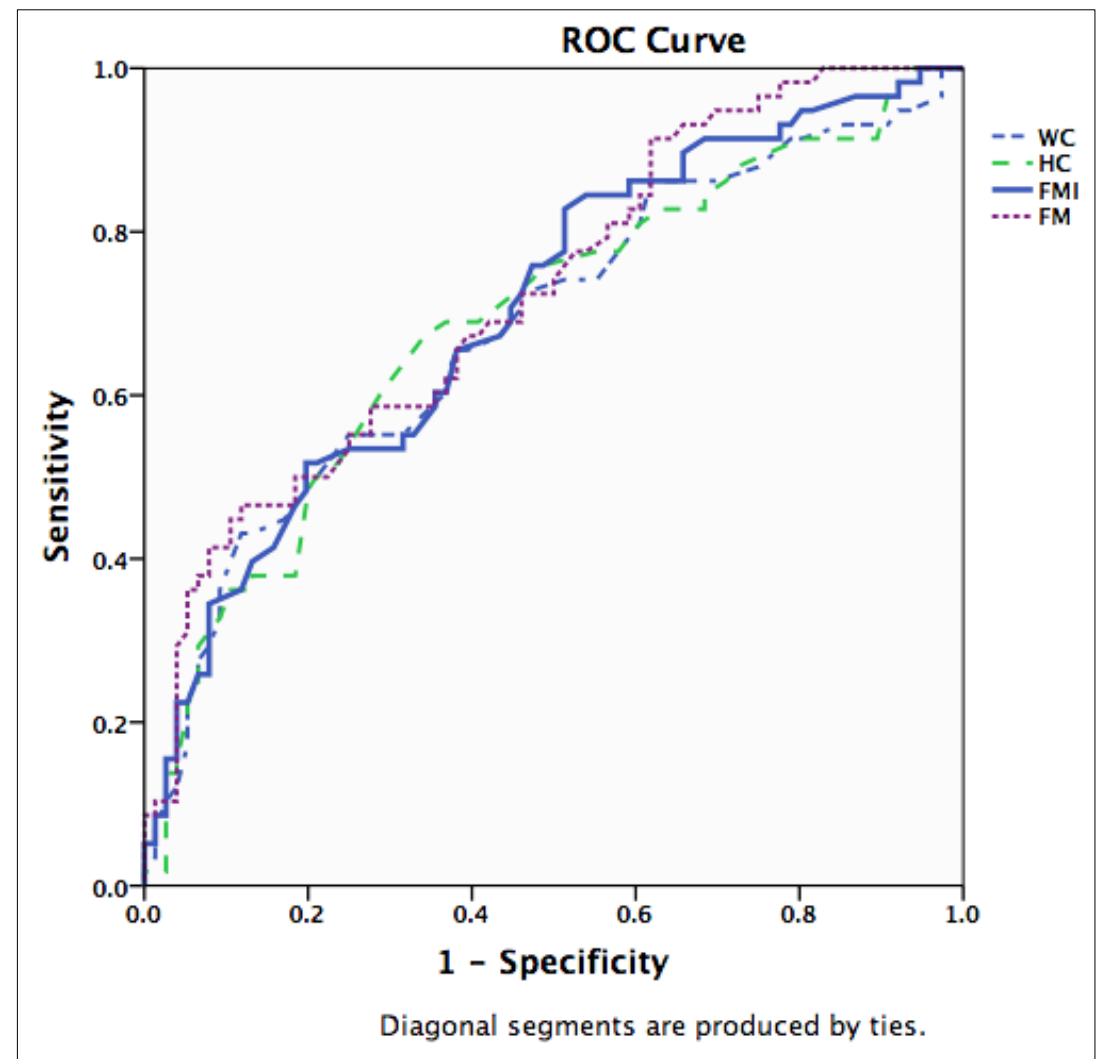

Area under the Curve: FM: 0.723, FMI: 0.703, WC: 0.684, HC: 0.690.

WC: Waist circumference, HC: Hip circumference, FM: Fat mass, FMI: Fat mass index

Figure 2. ROC curve analysis of fat mass, fat mass index and indirect methods according to metabolic syndrome 


\section{Discussion}

This study was demonstrated fatty liver a high frequency seen in obese children and it was more frequency seen in cases with MS. Anthropometric measurements and BIA results was moderately correlated in obese children. Different studies have reported the prevalence of fatty liver in obese children to range from $24 \%-58 \%$ [15, 16]. Although liver biopsy is the gold standard in the diagnosis of fatty liver, its invasive application prevents it from being used routinely as a diagnostic method [17]. The sensitivity and specificity of USG, which is a noninvasive and commonly used technique in the detection of fatty liver, were $89 \%$ and $93 \%$, respectively [18]. USG evaluation of fatty liver was positively related indirect measurement methods that was reported [19]. We evaluated fatty liver by USG, fatty liver was higher seen in cases with $>97^{\text {th }}$ percentile of FM, but fatty liver was not found in all of the patients with FM $>97^{\text {th }}$ percentile. The better correlation was found between FM, FMI with fatty liver in our study. According to MM didn't difference was found between obese children with and without fatty liver. Therefore the higher MM was found in cases with MS than without MS. Although higher fatty liver was detected in MS despite were found higher MM. These findings show that MS another important risk factor for fatty liver.

There isn't any previous study that evaluate of BIA and fatty liver in obese children. However, a positive correlation between fatty liver and UMAC and BMI has been demonstrated in the literature [20,21]. Adult study were demonstrated fatty composition and distribution especially abdominal fat accumulation was important for fatty liver and MS, therefore BIA records was better correlated with fatty liver than WC, BMI [22]. Better correlations were found between FM, FMI with fatty liver. Another study was reported that BIA FM positive correlated to BMI, WC in obese children [23]. Our findings support these data and FM, FMI moderately positive correlated to BMI-SDS, WC, UMAC and HC in obese children.

Fat mass accumulation, especially central fat deposition strongly associated of MS and cardiovascular risk [24]. Another study was reported fat accumulation was positive associated total cholesterol, triglyceride and LDL level while centrally deposited fat may influence the development of dyslipidemia more than the total body fat [25]. The study was reported the reduction of BMI-SDS was associated plasma lipid levels, but not accurately showed improved body composition. Those findings showed that using BMI-SDS method alone might not be enough in evaluation of obese children [25]. Our study was also demonstrated BMI-SDS no significant correlation with triglyceride and cholesterol levels. We found FM, FMI and UMAC significant correlation with triglyceride level.

Body mass index is often used in the diagnosis of obesity, but it does not differentiate between fat mass and fat-free mass and has a limited correlation with fat distribution [26]. Therefore, it is important to determine both fat tissue location and its percentage in body composition. The visceral location of fat tissue revealed in the developmental age is a significant factor in the development of metabolic syndrome [23]. Dual-energy X-ray absorptiometry is now accepted as a reference and gold standard method for assessing body composition in children and adolescents with obesity. It is expensive and accessibility is limited for most clinicians and practitioners [27]. Current findings indicate significant correlations between BIA and DEXA results in adults and adolescents [27, 28]. Indirect measurements methods give simple analysis in obese cases. Our findings show that there was better correlation FM and FMI with WC and HC in obese children.

MS is another problem in obese children. Waist size measurement is used as a diagnostic criterion for metabolic syndrome [12]. A positive correlation was determined between MS and FM, FMI, similarly positive correlation was also detected with HC and WC. In the NHANES study, DEXA analysis on 3004 participants, the area under curve (AUC) values of BMI and FMI showed were moderate identifiers for MS. Unlike our study, NHANES study had $16 \%$ obese participants and MS was identified in $6 \%$ of overall cases. The study population mean age was $16.1 \pm 2.51$, but our study population mean age was $13.3 \pm 2.2$, MS was defined in $43 \%$ of our study participants. This study showed that AUC values of FM and FMI higher than WC and BMI values for identifying of MS. The higher MM was found in cases with MS, also FM and FMI significantly higher in MS cases than without MS. FMI may be the better indicator for MS, because WC and BMI aren't demonstrating about body fat composition, especially it may also be affected by muscle mass in the cases.

The different type BIA models uses for measure of body composition. According to model, the measurement makes on the different position, example supine or standing position. Foot to foot BIA impedance is measured through the electronic pathway of the lower extremities Foot to foot BIA model conveniently predicts fat-free mass in Asian subjects was reported [29]. Another problem is validation of FM based on BIA model. We were used validated reference based on foot to foot model BIA in Turkish children [11]. Limitation of this method we didn't found about abdominal fat distribution, because it is important factor for fatty liver. But we found FM and FMI was moderately correlated with WC. 


\section{Conclusion}

Our results revealed that FM and FMI have a better correlated in obese children with fatty liver and metabolic syndrome than indirect measurement methods. However, the measurements by the foot-foot BIA method did not show a significant superiority in detecting fatty liver and metabolic syndrome in obese children according to indirect methods. The measure of FM, FMI, FM/MM were found the moderately positive correlation with BMI, UMAC, HC, and WC in obese children. The measurement of body FM and FMI can be used together with the indirect measurement methods to detect the fatty liver. FMI may be an alternative diagnostic criterion instead of WC for diagnosis of MS in children.

\section{Declaration of Competing Interest}

The authors declare that they have no known competing financial interests or personal relationships that could have appeared to influence the work reported in this paper.

\section{Ethical Approval}

All procedures performed in studies involving human participants were in accordance with the ethical standards of the institutional and/or national research committee and with the 1964 Helsinki declaration and its later amendments or comparable ethical standards. This study was approved by the local ethics committee (30.09.2014/18)7.

\section{References}

[1] McGill, H.C., McMahan, C.A., Gidding, S.S. (2008). Preventing heart disease in the 21st century: implications of the Pathobiological Determinants of Atherosclerosis in Youth (PDAY) study. Circulation, 117(9), 1216-1227. doi: 10.1161/CIRCULATIONAHA.107.717033.

[2] Dehghan, M., Merchant, A.T. (2008). Is bioelectrical impedance accurate for use in large epidemiological studies? Nutr J, 7, 26. doi: 10.1186/1475-2891-7-26.

[3] Deckelbaum, R. J., \& Williams, C. L. (2001). Childhood Obesity: The Health Issue. Obesity Research, 9(S11), 239S-243S. doi:10.1038/oby.2001.125.

[4] Fosbøl, M.Ø., Zerahn, B. (2015). Contemporary methods of body composition measurement. Clin Physiol Funct Imaging, 35 (2), 81-97. doi: 10.1111/cpf.12152

[5] Lazzer, S., Bedogni, G., Agosti, F., De Col, A., Mornati, D., Sartorio, A. (2008). Comparison of dual-energy X-ray absorptiometry, air displacement plethysmography and bioelectrical impedance analysis for the assessment of body composition in severely obese Caucasian children and adolescents. Br J Nutr,100(4), 918-924. doi: 10.1017/S0007114508922558.

[6] Alberti, G., Zimmet, P., Shaw, J., Bloomgarden, Z., Kaufman, F., Silink, M.; Consensus Workshop Group. (2004). Type 2 diabetes in the young: the evolving epidemic: the international diabetes federation consensus workshop. Diabetes Care 2004; 27(7): 17981811. doi: 10.2337/diacare.27.7.1798.

[7] Mok, E., Béghin, L., Gachon, P., Daubrosse, C., Fontan, J.E., Cuisset, J.M., Gottrand, F., Hankard, R. (2006). Estimating body composition in children with Duchenne muscular dystrophy: comparison of bioelectrical impedance analysis and skinfoldthickness measurement. Am J Clin Nutr, 83(1), 65-69. doi: 10.1093/ajcn/83.1.65.

[8] Bundak, R., Furman, A., Gunoz, H., Darendeliler, F., Bas, F., Neyzi, O. (2006). Body mass index references for Turkish children. Acta Paediatr, 95(2),194-198. doi: 10.1080/08035250500334738

[9] Elmaoğulları, S., Tepe, D., Uçaktürk, S.A., Karaca Kara, F., Demirel, F. (2015). Prevalence of Dyslipidemia and Associated Factors in Obese Children and Adolescents. J Clin Res Pediatr Endocrinol, 7(3), 228-234. doi: 10.4274/jcrpe.1867.

[10] Schutz, Y., Kyle, U.U., Pichard, C. (2002). Fat-free mass index and fat mass index percentiles in Caucasians aged 18-98 y. Int J Obes Relat Metab Disord, 26(7):953-960. doi: 10.1038/sj.ijo.0802037.

[11] Kurtoglu, S., Mazicioglu, M.M., Ozturk, A., Hatipoglu, N., Cicek, B., Ustunbas, H.B. (2010). Body fat reference curves for healthy Turkish children and adolescents. Eur J Pediatr,169,1329-1335. doi: 10.1007/s00431-010-1225-4.

[12] Zimmet, P., Alberti, K.G., Kaufman, F., Tajima, N., Silink, M., Arslanian, S., Wong, G., Bennett, P., Shaw, J., Caprio, S.; IDF Consensus Group. (2007). The metabolic syndrome in children and adolescents - an IDF consensus report. Pediatr Diabetes, 8(5): 299-306. doi: 10.1111/j.1399-5448.2007.00271.x.

[13] American Diabetes Association. Classification and diagnosis of diabetes. (2016). Standards of Medical Care in Diabetes. Diabetes Care, 39(1), 13-22. doi: 10.2337/dc16-er09.

[14] Kurtoğlu, S., Hatipoğlu, N., Mazicioğlu, M., Kendirici, M., Keskin, M., Kondolot, M. (2010). Insulin resistance in obese children and adolescents: HOMA-IR cut-off levels in the prepubertal and pubertal periods. J Clin Res Pediatr Endocrinol, 2(3),100-106. doi: $10.4274 /$ jcrpe.v2i3.100. 
[15] Kawasaki, T., Hashimoto, N., Kikuchi, T., Takahashi, H., Uchiyama, M. (1997). The relationship between fatty liver and hyperinsulinemia in obese Japanese children. J Pediatr Gastroenterol Nutr, 24(3), 317-321. doi: 10.1097/00005176-19970300000015.

[16] Erol, M., Bostan Gayret, O., Tekin Nacaroglu, H., Yigit, O., Zengi, O., Salih Akkurt, M., Tasdemir, M. (2016). Association of Osteoprotegerin with Obesity, Insulin Resistance and Non-Alcoholic Fatty Liver Disease in Children. Iran Red Crescent Med J, 18 (11), e41873. doi: 10.5812/ircmj.41873.

[17] Marzuillo,. P., Grandone, A., Perrone, L., Miraglia Del Giudice, E. (2015). Controversy in the diagnosis of pediatric nonalcoholic fatty liver disease. World J Gastroenterol, 21(21) 6444-6450. doi: 10.3748/wjg.v21.i21.6444.

[18] Joseph, A.E., Saverymuttu, S.H., Al-Sam, S., Cook. M.G., Maxwell, J.D. (1991). Comparison of liver histology with ultrasonography in assessing diffuse parenchymal liver disease. Clin Radiol, 43(1),26-31. doi: 10.1016/s0009-9260(05)803502 .

[19] Chiloiro, M., Riezzo, G., Chiarappa, S., Correale, M., Guerra, V., Amati, L., Noviello, M.R., Jirillo, E. (2008). Relationship among fatty liver, adipose tissue distribution and metabolic profile in moderately obese children: an ultrasonographic study. Curr Pharm Des, 14 (2), 2693-2698. doi: 10.2174/138161208786264197.

[20] Chan, D.F., Li, A.M., Chu, W.C., Chan, M.H., Wong, E.M., Liu, E.K., Chan, I.H., Yin, J., Lam, C.W., Fok, T.F., Nelson, E.A. (2004).Hepatic steatosis in obese Chinese children. Int J Obes Relat Metab Disord, 28(10), 1257-1263. doi: 10.1038/sj.ijo.0802734.

[21] Pang, Q., Zhang, J.Y., Song, S.D., Qu, K., Xu, X.S., Liu, S.S., Liu, C. (2015). Central obesity and nonalcoholic fatty liver disease risk after adjusting for body mass index. World J Gastroenterol, 21(5), 1650-1662. doi: 10.3748/wjg.v21.i5.1650.

[22] Vitturi, N., Soattin, M., De Stefano, F., Vianello, D., Zambon, A., Plebani, M., Busetto, L. (2015). Ultrasound, anthropometry and bioimpedance: a comparison in predicting fat deposition in non-alcoholic fatty liver disease. Eat Weight Disord, 20(2), 241247. doi: 10.1007/s40519-014-0146-z.

[23] Goluch-Koniuszy, Z.S., Kuchlewska, M. (2017). Body composition in 13-year-old adolescents with abdominal obesity,depending on the BMI value. Adv Clin Exp Med, 26(6):973-979. doi: 10.17219/acem/61613.

[24] Botton, J., Heude, B., Kettaneh, A., Borys,. J.M., Lommez, A., Bresson, J.L., Ducimetiere, P., Charles, M.A.; FLVS Study Group. (2007). Cardiovascular risk factor levels and their relationships with overweight and fat distribution in children: The Fleurbaix Laventie Ville Sante’ II study. Metabolism, 56(5), 614-622. doi:10.1016/j.metabol.2006.12.006.

[25] Nielsen, T.R.H., Fonvig, C.E., Dahl, M., Mollerup, P.M., Lausten-Thomsen, U., Pedersen, O., Hansen, T., Holm, J.C. (2018). Childhood obesity treatment; Effects on BMI SDS, body composition, and fasting plasma lipid concentrations. PLoS One, 13(2):e0190576. doi: 10.1371/journal.pone.0190576.

[26] Verney, J., Metz, L., Chaplais, E., Cardenoux, C., Pereira, B., Thivel, D. (2016). Bioelectrical impedance is an accurate method to assess body composition in obese but not severely obese adolescents. Nutr Res, 36(7), 663-70. doi: 10.1016/j.nutres.2016.04.003.

[27] Sweeting, H.N. (2008). Gendered dimensions of obesity in childhood and adolescence. Nutr J, 7,1-14. doi: 10.1186/1475-2891$7-1$.

[28] Wan, C.S., Ward, L.C., Halim, J., Gow, M.L., Ho, M., Briody, J.N., Leung, K., Cowell. C.T., Garnett, S.P. (2014). Bioelectrical impedance analysis to estimate body composition, and change in adiposity, in overweight and obese adolescents: comparison with dual-energy x-ray absorptiometry. BMC Pediatr, 14,249. doi: 10.1186/1471-2431-14-249.

[29] Wu, C.S., Chen, Y.Y., Chuang, C.L., Chiang, L.M., Dwyer, G.B., Hsu, Y.L., Huang, A.C., Lai, C.L., Hsieh, K.C. (2015). Predicting body composition using foot-to-foot bioelectrical impedance analysis in healthy Asian individuals. Nutr J, 14:52. doi: 10.1186/s12937-015-0041-0. 